\title{
Ovarian Thecoma Fibroma Causing Menstrual Disorder \& Infertility: Dramatic Resolution with Surgery
}

\author{
F. C. J. Emegoakor ${ }^{1}$, P. C. Udealor ${ }^{1}$, E. P. Ezenkwele', M. Nzegwu' ${ }^{2}$ \\ ${ }^{1}$ Department of Obs. and Gyn., University of Nigeria, Ituku Ozalla Campus, Enugu State, Nigeria \\ ${ }^{2}$ Department of Histopathology, University of Nigeria Teaching Hospital Ituku Ozalla, Enugu State, Nigeria \\ Email: ^faustachioma.emegoakor@unn.edu.ng
}

How to cite this paper: Emegoakor, F.C.J., Udealor, P.C., Ezenkwele, E.P. and Nzegwu, M. (2020) Ovarian Thecoma Fibroma Causing Menstrual Disorder \& Infertility: Dramatic Resolution with Surgery. Open Journal of Obstetrics and Gynecology, 10, 815-819.

https://doi.org/10.4236/ojog.2020.1060076

Received: April 13, 2019

Accepted: June 19, 2020

Published: June 22, 2020

Copyright $\odot 2020$ by author(s) and Scientific Research Publishing Inc. This work is licensed under the Creative Commons Attribution International License (CC BY 4.0).

http://creativecommons.org/licenses/by/4.0/

\begin{abstract}
Purpose: To report the case of successfully managed oligo-amenorrhoea with infertility as a result of thecoma fibroma in a young Nigerian Igbo woman. Case Presentation: She was a 24-year-old, married, nulliparous woman who presented with 5 years history of oligomenorrhoea and 18 months history of infertility. Following her menarche at 14 years of age, she had a regular 30-day menstrual cycle with 4 - 6 days moderate flow. Symptoms of oligomenorrhoea worsened to amenorrhoea over time with menstrual flow occurring only when a progesterone agent was used. Clinical evaluation revealed no abnormalities in the systems apart from the presence of left adnexal mass on pelvic examination. Radiological test suggested benign left adnexal mass. Results: She had surgical removal of the mass after work up. Her normal menstrual flow resumed 48 hours following surgery and became regular thereafter. The histopathology report confirmed thecoma-fibroma. She commenced sexual intercourse 12 weeks after surgery. At 18 weeks post operative period, she presented with amenorrhoea and pregnancy symptoms. Intra-uterine pregnancy was confirmed with a pelvic ultrasound scan. She was managed in antenatal clinic till 38 weeks gestational age when she had spontaneous vaginal delivery of a live female neonate that weighed $3 \mathrm{~kg}$ with good APGAR scores. She is currently being followed up. Conclusion: Ovarian thecoma-fibroma is a rare cause of menstrual irregularities and infertility in young women. Surgical removal could result in a dramatic and favourable clinical response, as in the case presented.
\end{abstract}

\section{Keywords}

Thecoma-Fibroma, Oligo-Amenorrhoea, Infertility, Premenopausal 


\section{Introduction}

Ovarian thecoma-fibroma is one of spectrums of benign sex cord stromal ovarian tumours found more in the post menopausal women [1] [2] [3]. It has a wide range of clinical manifestations due to various factors but can also be asymptomatic [1]. Though documented as being uncommon in occurrence [2] [3], ovarian thecoma-fibroma is even rarer in young women. We report one of such rare cases in a young nulliparous woman who had features of oligo-amenorrhoea and infertility.

\section{Case Summary}

She was a 24-year-old married woman who presented with 5 years history of oligomenorrhoea and 18 months history of infertility. Following her menarche at 14 years of age, she had a regular 30-day menstrual cycle with 4 - 6 days moderate flow. Symptoms of oligomenorrhoea developed after 5 years and worsened to amenorrhoea over time with menstrual flow occurring only when a progesterone agent was used. She had no significant past gynaecological history and did not take any other hormonal drugs prior to onset of symptoms. She had no other systemic symptoms.

Clinical examination revealed no abnormalities in the systems apart from the presence of left adnexal mass on pelvic examination. Her body mass index was $22 \mathrm{~kg} / \mathrm{m}^{2}$. She had no features suggestive of excessive androgen. Hormone profile was essentially normal and included FSH-7.362 mIU/ml; LH-6.857 mIU/ml; PRL-8.022 ng/ml; TSH-1.6 mIU/L; Testosterone-28.024 ng/dl; DHEAS 12.6 $\mathrm{ng} / \mathrm{ml}$.

Pelvic ultrasound revealed a solid, eccentrically located mass in the left ovary. The mass measured about $9 \mathrm{~cm} \times 7 \mathrm{~cm} \times 4 \mathrm{~cm}$, was well outlined, hypoechoic and with minimal Doppler flow signals. There was no ascites and other pelvic structures were normal. Chest X-ray and hysterosalpingogram showed normal findings.

She was counseled and consent obtained for surgical removal of the ovarian mass. Intra operative findings were a well circumscribed but lobular, firm and pale-yellowish mass measuring about $10 \mathrm{~cm} \times 8 \mathrm{~cm} \times 5 \mathrm{~cm}$ in the left ovary. The right ovary and other pelvic structures were normal. There was no ascites. Peritoneal washing was done and specimen sent for cytology while the mass was sent for histology. 48 hours post surgery, she started having menstrual flow which was normal and lasted for 6 days.

The histopathology report read thus: Sections show large rounded ill-defined cells with moderate abundant sometimes clear/vacuolated cytoplasm containing lipids admixed or separated with vague moderate amounts of fibrous tissues and consistent with thecoma-fibroma.

Cytology of peritoneal fluid yielded no abnormal finding. Patient was discharged on the 5th post operative day and given a 6-week appointment. During this first follow up visit she had seen her normal menses. At 12 weeks post sur- 
gery, she had resumed sexual activities and was on her $4^{\text {th }}$ menstrual cycle following surgery. At 18 weeks post operative period, she presented with amenorrhoea, weakness and nausea. Serum pregnancy test and pelvic ultrasound scan done confirmed early pregnancy. She was managed in antenatal clinic till 38 weeks gestational age when she had spontaneous vaginal delivery of a live female neonate that weighed $3 \mathrm{~kg}$ with good APGAR scores. She is currently being followed up.

\section{Discussion}

This case is reported to highlight the possibility of ovarian thecoma fibroma as a differential diagnosis of menstrual irregularities and even infertility in young women. Its successful management as reported is intended to add to the existing literature to serve as reference management protocol of such cases in order to improve clinical practice and training.

Ovarian thecoma-fibroma is one of the benign variants of sex cord stromal tumours which affect women infrequently [4] [5]. Its clinical presentation is variable, non-specific [2] and may be influenced by its size, endocrinological activity, histological composition, patients' age and other peculiar factors. Thus, it may be described as having a wide spectrum of presentation in clinical settings [1] [6]. The index patient had no specific symptoms with only menstrual anomaly and infertility being her chief complaints. Incidental clinical pelvic examination finding of an adnexal mass gave the key clue to a possible ovarian pathology. Her ultrasound findings were similar to earlier documentations by other authors [1] [4]. Patients with ovarian thecoma-fibroma who have menstrual disorder may demonstrate derangement in hormone profile [4] [7] while others may have values within normal limits of the reproductive hormones as demonstrated in the index patient. Findings from other ancillary investigations to exclude differential diagnoses or associated syndromes were in keeping with benign ovarian tumour in our patient. Though some authors reported raised serum level of CA 125 in association with ovarian fibro-thecomas, [1] [6] this marker is said to have low specificity for ovarian pathologies especially in premenopausal women [8]. Also, Numanoglu et al. [9] concluded in their work that there are no markers that can give precise diagnosis of ovarian fibro$\mathrm{ma} /$ thecoma-fibroma prior to surgery. The treatment is usually individualized but surgical removal of ovarian mass, histopathology and follow up have been commonly reported [2] [3] [6] [7] [10]. Clinical recovery usually follows this line of management for the symptomatic patients [1] [2] [3] [6] [7]. We noticed a quick resolution of secondary amenorrhoea within 48 hours after surgery; this is shorter than the time interval reported by Meyer et al. [7] for reversal of secondary amenorrhoea following surgery for ovarian thecoma in their own case report. Cases of successful pregnancies and deliveries one year following surgery have been documented [1] and are in consonance with findings in our patient who conceived soon after she resumed sexual activity, 12 post operative weeks. She also had spontaneous vaginal delivery of a normal term baby. 


\section{Conclusion}

Ovarian thecoma-fibroma, though uncommon in young premenopausal women, is an important cause of oligomenorrhea, secondary amenorrhea and or infertility. Careful clinical evaluation is therefore paramount in distinguishing this condition from other differential diagnoses. Surgical option of management, histology of surgical specimen and keen follow-up are recommended for optimal care.

\section{Disclosures}

1) Informed consent was given by the patient to permit the authors to communicate her case/condition to wider audience in both electronic and print media.

2) I hereby declare that this manuscript, prior to its submission for peer review was presented as an e-poster during the International Federation of Gynecology and Obstetrics (FIGO) XXII FIGO World Congress 2018 at RioCentro, Brazil.

3) The authors report no conflicts of interest in this work.

\section{Conflicts of Interest}

The authors declare no conflicts of interest regarding the publication of this paper.

\section{References}

[1] Chen, H., Liu, Y., Shen, L.F., Jiang, M.J., Yang, Z.F. and Fang, G.P. (2016) Ovarian the Coma-Fibroma Groups: Clinical and Sonographic Features with Pathological Comparison. Journal of Ovarian Research, 9, 81. https://doi.org/10.1186/s13048-016-0291-2

[2] Chechia, A., Attia, L., Ben Temime, R., et al. (2008) Incidence, Clinical Analysis, and Management of Ovarian Fibromas and Fibrothecomas. American Journal of Obstetrics \& Gynecology, 199, 473.e1-473.e4. https://doi.org/10.1016/j.ajog.2008.03.053

[3] Leung, S.W. and Yuen, P.M. (2006) Ovarian Fibroma: A Review on the Clinical Characteristics, Diagnostic Difficulties, and Management Options of 23 Cases. Gynecologic and Obstetric Investigation, 62, 1-6. https://doi.org/10.1159/000091679

[4] Horta, M. and Margarida, T. (2015) Cunha Sex Cord-Stromal Tumors of the Ovary: A Comprehensive Review and Update for Radiologist. Diagnostic and Interventional Radiology, 21, 277-286. https://doi.org/10.5152/dir.2015.34414

[5] Haroon, S., Zia, A., Idrees, R., Memon, A., Fatima, S. and Kayani, N. (2013) Clinicopathological Spectrum of Ovarian Sex Cord-Stromal Tumors; 20 Years' Retrospective Study in a Developing Country. Journal of Ovarian Research, 6, 87. https://doi.org/10.1186/1757-2215-6-87

[6] Dhull, A.K., Kaushal, V., Mathur, S. and Agarwal, R. (2011) Fibrothecoma of Ovary: A Rare Case Presentation. The Internet Journal of Third World Medicine, 9, $10.5580 / 16 \mathrm{c} 2$.

https://www.researchgate.net/publication/274039409 Fibrothecoma of Ovary-_A Rare Case Presentation 
[7] Meyer, A.C., Papadimitriou, J.C., Silverberg, S.G. and Sharara, F.I. (2000) Secondary Amenorrhea and Infertility Caused by an Inhibin-B-Producing Ovarian Fibrothecoma. Fertility and Sterility, 73, 258-260.

https://doi.org/10.1016/S0015-0282(99)00511-7

[8] Foti, P.V., Attinà, G., Spadola, S., Caltabiano, R., Farina, R., Palmucci, S., Zarbo, G., Zarbo, R., D’Arrigo, M., Milone, P. and Ettorre, G.C. (2016) MR Imaging of Ovarian Masses: Classification and Differential Diagnosis. Insights Imaging, 7, 21-41. https://doi.org/10.1007/s13244-015-0455-4

[9] Numanoglu, C., Kuru, O., Sakinci, M. and Ulker, A.O. (2013) Ovarian Fibroma/Fibrothecoma: Retrospective Cohort Study Shows Limited Value of Risk of Malignancy Index Score. The Australian and New Zealand Journal of Obstetrics and Gynaecology, 53, 287-292. https://doi.org/10.1111/ajo.12090

[10] Mawad, N.M. and Hassanein, O.M. (1994) Ovarian Fibro-Thecoma in a 19 Years Old Sudanese Girl. Gynaecological Case Report. Clinical and Experimental Obstetrics and Gynecology, 21, 243-245.

\section{Abbreviations}

CA 125: Cancer antigen 125;

DHEAS: Dehydroepiandrosterone sulfate;

FSH: Follicle stimulating hormone;

LH: Leutenising hormone;

PRL: Prolactin;

TSH: Thyroid stimulating hormone. 